\title{
Analysis on Psychological Crisis Prevention of College Students Based on Classification of Localization
}

\author{
Tusheng Jiang \\ College of political Sciences, Chongqing Normal University, Chongqing, 401331, China
}

Keywords: Localization; psychological crisis prevention; college students

\begin{abstract}
It is necessary to develop psychological crisis intervention of college students because it can promote the students' psychological health and to have better growth. However, the psychological crisis intervention in China mainly takes examples from the west during research in idea, mode and other aspects, without strict classification of localization, thus the operation of psychological crisis intervention in China has an "endemic" problem. Therefore, it is necessary to explore the psychological crisis intervention based on classification of localization. Based on this, the psychological crisis intervention of college students based on classification of localization is analyzed and discussed in this paper.
\end{abstract}

\section{Introduction}

The psychological crisis, from the psychological world of the United States, is a diversified concept and emphasizes the negative psychological state of concerned person on an event or situation, under which the individual's cognition, emotion and behavior are imbalanced seriously. As a college student, he/she may suffer from stress of family, school and society, thus generating certain psychological crisis. To promote the healthy growth of college students, it is necessary to conduct psychological crisis intervention of college students. Therefore, by virtue of the psychological crisis intervention in foreign countries, the psychological crisis intervention has classification of localization to make it adjust the psychological crisis of college students, which will be conducive to the healthy growth and better development of college students in the future.

\section{Differences of psychological crisis intervention between the east and the west}

(I) Difference of cultural background

Both the judgment, explanation and intervention method and process of psychological crisis will be affected by the cultural concept of intervenor. From the current use of psychological crisis intervention, the psychological crisis intervention with culture of North America and Europe as the background is widely used. However, due to the great cultural difference between the east and the west, the cultural difference of psychological crisis intervention between the east and the west should be known first for the purpose of referring to the psychological crisis intervention experiment based on the cultural background of North America and Europe.

(1) Difference of judgment and explanation of crisis state. In the western culture, the rights and privacy of individuals are respected and the emotions of individuals must be allowed and will not be affected by certain factor though the individuals belong to the society. Therefore, the crisis intervention pays more attention to closed and professional intervention. It is not the same in the eastern culture, in which the self-sacrifice is advocated, that is, the interests of individuals can be sacrificed for the sake of the state. In this case, the judgment and explanation of crisis intervention pay more attention to the collective significance and value.

(2) Difference of psychological identification of audiences. The western culture stresses the independence value of individuals. In case of any psychological crisis, individuals often seek help from professional institute and get normal services by paying certain money in order to solve their psychological crisis. Affected by the western culture, people can treat the psychological crisis objectively and accept the psychological assistance. However, affected by the traditional culture for a long time, Chinese people think psychological crisis is a defect and unacceptable. This indicates 
that Chinese people have psychological cognitive defect, so the degree of corresponding psychological crisis intervention is poor.

(3) Difference of assistance means. Restrained by western inherent system and affected by strong independence of westerners, westerners can accept the professional psychological institution and will consult and receive treatment in the professional institution in case of any psychological crisis. However, affected by the cultural environment, easterners are willing to receive treatment or help from familiar people or in the familiar environment, rather than independent professional consultative institution.

(II) Difference of intervention system structure

For the development of psychological crisis intervention with the culture of North America and Europe as the background, the western psychological crisis intervention has formed a relatively complete psychological crisis intervention mechanism, whose establishment gets supports from government, volunteers and professionals, thus greatly improving the western psychological crisis intervention effect. It can be said that the western psychological crisis intervention has transited from civilian donation to governmental support, thus making the psychological crisis intervention normalized, standardized and professional. However, for the development of psychological crisis intervention in China, the psychological crisis intervention, in an initial stage, develops to a certain degree only in colleges and other partial social institution and does not develop into a large scale in the society. At present, the psychological crisis intervention in China seriously lacks of government investment, volunteer support and professional support, thus the psychological crisis intervention system is hard to build.

\section{Main performance of psychological crisis of college students}

(I) Environmental adaptation problem

Freshmen often have psychological crisis due to the environmental adaptation problem. The students should accept new environment, lifestyle, learning style, communication mode and psychological style from high school to college, which make them unadapted. In this case, college students easily have psychological crisis due to vulnerable psychology.

(II) Learning style problem

The tense study arrangement and teacher's urge in high school make students develop passive arrangement and learning habits. However, the learning style in college is different, in which students are required to arrange their learning, allocate their learning time and study independently, which are not applicable to students with poor self-control ability. As students cannot arrange their learning progress and conduct self learning independently, they will get frustrated upon learning, thus leading to unhealthy emotions. As time passes, students may have certain psychological crisis.

(III) Interpersonal relationship problem

In college, the inharmonious interpersonal relationship is often the main cause for psychological crisis of college students. Compared with the interpersonal relationship in high school, the interpersonal relationship in college is more complex. Due to the different ways of treating people and personality characteristics of individuals, college students often have various problems in the interpersonal communication, which may make college students feel uneasy, tense, lonely and depressive, thus promoting the generation of psychological crisis.

\section{Prominent problems in psychological crisis intervention of college students}

From the overall situation of the psychological crisis intervention of college students in China, the psychological crisis intervention of college students has the following prominent problems:

(I) Subject problem of psychological crisis intervention

The psychological crisis intervention pays attention to the self-control of concerned person and the psychological crisis can be gradually improved by the intervention of professional until rehabilitation. However, Chinese colleges only stress the active role of others and neglect the self-control of intervention object, which is hard to play an intervention role. The subject of 
psychological crisis intervention is not clear and the intervention object only receives the intervention of other passively, which may lead to two results finally: first, the psychological crisis of intervention object will occur over and over; second, the intervention object will have serious psychological problem finally. Therefore, attention must be paid to the subject problem of psychological crisis intervention.

(II) System engineering problem of psychological crisis intervention

The psychological crisis intervention of college students is a project engineering, whose operation needs the supports of all sectors. However, there is no perfect psychological crisis intervention system in most of Chinese college mainly due to the low investment, lacking of professional and insufficient social supports, thus the construction of psychological crisis intervention system is hard.

(III) Poor psychological crisis intervention ability of colleges

As the psychological crisis intervention in China is in an initial age and mainly refers to the psychological crisis intervention experiment in foreign countries and the psychological crisis intervention consciousness is inadequate, the psychological crisis intervention in China has many problems. Although Chinese colleges have been devoted to psychological crisis intervention for recent years, but no good effects are obtained.

\section{Analysis of psychological crisis intervention of college students based on classification of localization}

(I) Build effective timely intervention response mechanism

The psychological crisis intervention in foreign countries has been professional with a scale, but there is no perfect psychological crisis intervention system in China, so Chinese colleges should build the effective timely intervention response mechanism based on the cultural background in China and combined the actual situation to restrain the implementation of psychological crisis intervention by such mechanism. As a special and important content of psychological health education, the psychological crisis intervention should be separated from the ideological and political education and taken as a course of enhancing students' psychological health. In addition, it is required to develop the psychological crisis intervention concept and mode to conduct psychological intervention of students according to the content of timely intervention response mechanism. Of course, for this purpose, the supports of student work system professionals are needed. Therefore, the proper psychological health knowledge and skill training, organization of student work system personnel's participation in training and improvement of professional level of system personnel can promote the implementation of local psychological crisis intervention in college to a certain degree.

(II) Objective knowledge and positive prevention

The psychological crisis prevention is an important work for Chinese colleges. At present, the psychological crisis prevention in Chinese colleges mainly includes psychological health education and psychological census.

(1) Psychological health education

The psychological health education is a prevention to effectively reduce the psychological crisis of college students and its contents are mainly psychological health knowledge and psychological quality education, which promotes students to know what's the psychological health and how to be psychologically healthy by psychological adjustment. However, it must be noted that as the psychological crisis of students is closely related to the living environment, emotion, event and other aspects, the individual will easily have psychological crisis in case of any great influence. It can be said that the psychological health education knowledge is just universal and basic psychological crisis prevention.

(2) Psychological census

The colleges expect to find the theoretical assumption of potential crisis personnel by psychological census of students that the psychological crisis exists in psychological crisis-prone population and the psychological response characteristics of psychological crisis-prone population, 
especially the inherent personality basis and special response method, are screened by census. However, is the psychological census of colleges really effective? In view of this problem, the author knows by looking up some information and carrying out census of college students in Panzhihua with UPI and SCL-90 that the psychological census cannot completely and accurately screen out the person of psychological crisis. Of course, this does not mean that the psychological census is meaningless because the psychological census can find students with the possibility of psychological crisis. Therefore, the timely intervention of college students by psychological census can reduce the possibility of psychological crisis of college students.

From the experience of western psychological crisis intervention, the psychological crisis intervention has many methods, but which one is suitable for China is to be determined by conducting proper and effective psychological crisis defense combined with the cultural features of China and actuation situation of colleges. Thus, it can reduce the possibility of psychological crisis of college students in China as far as possible.

(III) Perfect the delay intervention

The focus of psychological crisis intervention is to find the proper and effective coping skills and environmental support resources quickly in intervention to help the concerned people go out from the psychological crisis. Therefore, it is important to continuously perfect the delay intervention. The timely intervention of psychological crisis person can stabilize such person's emotion and behavior and the effective delay intervention at this time can effectively help the crisis person conduct psychological regulation and relieve the psychological crisis, thus reducing the possibility of psychological crisis recurrence. In addition, it is also conducive to help the psychological crisis person go out of psychological crisis and grow healthily. So, how to perfect the delay intervention? The establishment of psychological crisis intervention subsequent support system is an effective measure. After establishing an subsequent support system combined the psychological health education personnel and social resources, the concerned person can be further treated by psychological counseling professional after receiving timely intervention and get more help and supports, thus recovering ASAP.

\section{Conclusion:}

The psychological crisis brings great harmful influences on college students and hinders the healthy growth and sound development of college students to a certain degree. As the cradle of cultivating excellent talents, colleges should pay attention to enhance the psychological crisis intervention of college students. The timely psychological crisis intervention of college students in China can be conducted by reference to the relevant contents of psychological crisis intervention in foreign countries and then localizing such intervention to form the local psychological crisis intervention. The delay intervention and subsequent intervention can reduce the psychological crisis of college students, promote the healthy growth of students and make them become the excellent talents required by the state.

\section{Acknowledgments}

This paper is the phased achievement of 2014 Key humanities \& Social Science Project of Chongqing Municipal Commission of Education, Research on Psychological Crisis Intervention of College Students Based on Classification of Localization.

\section{References}

[1] Li Zizhang. Establishment of Psychological Crisis Warning and Intervention Mechanism of Higher Vocational College Students[J]. Campus Life \& Mental Health, 2014(04).

[2] Dai Jun. Discussion on Localization of Psychological Crisis Intervention Mechanism of College Students[J]. Ideological \& Theoretical Education, 2008(13). 
[3] Wang Jianguo. Research on Theoretical Origin and Strategy of Psychological Crisis Intervention of College Students[J]. Journal of Southwest Agricultural University (Social Science), 2007(03).

[4] Song Ge. On Psychological Crisis Prevention of College Students[A]. Proceedings of 2011 Beijing University Psychological Quality Education Conference and Beijing University Psychological Quality Education Theory and Practice Seminar[C].2012.

[5] Li Beida, Liu Ruiqing, Li Zhiqiang, Zhou Xiuyun. On Influence of Informal Supporting System of College Students on Their Psychological Heath State —-Taking Nine Colleges in Nanjing as Examples[J]. Rural Economy and Science-Technology, 2008(05).

[6] Song Zhenshao. Origin of Psychological Crisis of Colleges Students and Its Education Strategy[A]. Proceedings of Psychological Health Education Progress in Colleges_-The 10th National University Psychological Health Education and Psychological Counseling Symposium[C].2007.

[7] Zha Jing, Liu Haihua. Thinking and Try of Psychological Crisis Intervention in Colleges[A]. Proceedings of Psychological Health Education Progress in Colleges-The 10th National University Psychological Health Education and Psychological Counseling Symposium[C].2007.

[8] Li Jun. Research Summary of Psychological Crisis of College Students and Its Intervention[J]. Journal of Chifeng University (Philosophy and Social Science),2006(06).

[9] Guo Hui, Li Mandi, Su Meng. Give Full Play to Peer Power and Help Psychological Quality Education of College Students_-Taking Beijing University of Chinese Medicine as an Example[A]. Proceedings of 2011 Beijing University Psychological Quality Education Conference and Beijing University Psychological Quality Education Theory and Practice Seminar[C],2012. 